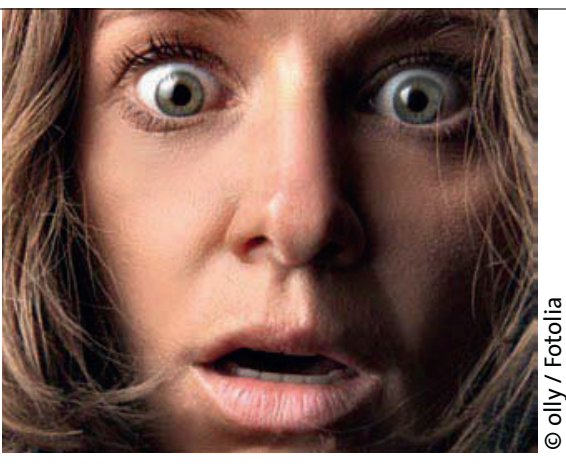

rungen der Herzfrequenz und des Blutdrucks. Probanden, die während ihrer Rede Angst oder Ärger verspürt hatten, reagierten aber mit einem signifikanten Anstieg von IL-6. Dieser Mechanismus könnte das Risiko für enzündliche Erkrankungen erhöhen. Kurt Malberg

Carroll JE et al. Access Online 2011; 25: 232-8

\section{KURZ GEMELDET}

Allergy News

\section{Psychostress und IL-6}

E orscher der University of Pittsburgh, USA, haben bei 102 Gesunden ein akutes Stressprotokoll durchgeführt. Der Stress wurde ausgelöst, indem man die Probanden vor einer Jury bei laufender Kamera eine Rede halten ließ. Nach einer einleitenden 30-minütigen Ruhephase, einer fünfminütigen Rede und einer erneuten, abschließenden 30-minütigen Ruhephase wurden jeweils Blutspiegel des proinflammatorischen Zytokins Interleukin-6 (IL-6) bestimmt. Nach der Rede wurden die Probanden gefragt, wie sie sich fühlten. Das IL-6 verhielt sich individuell sehr unterschiedlich. Die Konzentration war nicht assoziiert mit Verände-

\section{Kamikazebehandlung}

1 anche Menschen legen sich auf eine Sonnenbank, weil sie sich die Linderung ihres Hautleidens versprechen. Doch kann diese Art der Selbstbehandlung lebensgefährlich werden, warnen Dermatologen der Indiana University School of Medicine: Eine Patientin hatte nach der Einnahme von Ibuprofen einen leichten Hautausschlag bekommen. Nach einer Selbstbehandlung im Sonnenstudio entwickelte sie eine toxische epidermale Nekrolyse (TEN), mit schwerer Blasenbildung und Blutdruckabfall. TEN führt in $20 \%$ der Fälle zum Multiorganversagen und zum Tod.

Dirk Einecke

Gatson T et al. Arch Dermatol 2011 Feb 21; DOI: 10.1001/archdermatol.2011.13

\section{„Statussymbol“ malignes Melanom}

\begin{abstract}
e höher der sozioökonomische Status einer Frau, desto höher ist ihr Risiko für ein malignes Melanom. Diesen überraschenden Zusammenhang ergab eine Langzeitstudie des California Cancer Registry, USA, auf der Basis des kalifornischen Krebsregisters. Die Untersuchung berücksichtigte die Daten von 3.800 weißhäutigen Frauen und Mädchen im Alter zwischen 15 und 39 Jahren, bei denen 3.842 Melanome diagnos-
\end{abstract}

tiziert worden waren. Wohlsituierte Frauen hatten danach ein fast sechsfach höheres Risiko für ein malignes Melanom als Frauen, die in ärmlichen Verhältnissen lebten. Frauen aus noblen Wohngegenden reagierten überdies besonders empfindlich auf eine hohe UVBelastung.

Dirk Einecke

Hausauer AK et al. Arch Dermatol 2011 Mar 21; DOI: 10.1001/archdermatol.2011.44

\section{Viele Wiederholungstäter bei Arzneimittelallergie}

\footnotetext{
eder dritte Arzneimittelallergiker nimmt einige Jahre nach der Diagnose wieder ein Medikament ein, dass das auslösende Allergen enthält, haben Allergologen von der Hautklinik Göttingen festgestellt. Die Forscher hatten 80 Patienten mit einer Allergie gegen nicht steroidale Schmerzmittel wie Acetylsalicylsäure, Paracetamol, Diclofenac oder Ibuprofen zu ihren Erfahrungen einige Jahre nach der Diagnose befragt. $41 \%$ hatten trotz der bekannten Allergie erneut das auslösende Medikament eingenommen. Die Gründe dafür variierten: Ein Teil der Patienten hatte vergessen, auf welchen Stoff er allergisch reagiert. $\odot$ 127:284-5
}

\section{Laute Keimschleudern}

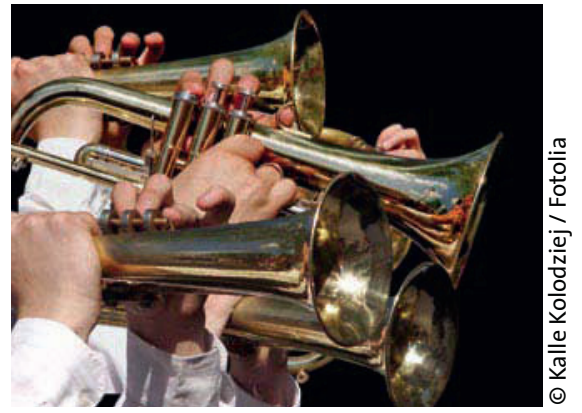

Us S-Forscher haben die Blasinstrumente einer Highschool-Band bakteriologisch untersucht. Sechs von 13 Instrumenten waren in der Woche zuvor nat lang unbenutzt geblieben. Die Forscher fanden 442 unterschiedliche Bakterien - überwiegend Staphylokokken, 58 verschiedene Schimmelpilze und 19 Hefepilze. Viele Bakterien waren hoch resistent gegen die üblichen Antibiotika. Fazit: Blasinstrumente sollten nach jedem Spiel desinfiziert werden. Dabei genügt es nicht, das Mundstück zu säubern. Die Keime waren über das ganze Instrument verteilt.

Dirk Einecke gespielt worden, sieben waren einen Mo- 